\title{
Numerical Simulation of Laser Fusion Zone Profile of Lotus-Type Porous Metals
}

\author{
Takuya Tsumura ${ }^{1}$, Taichi Murakami ${ }^{2}$, Hideo Nakajima $^{3}$ and Kazuhiro Nakata ${ }^{1}$ \\ ${ }^{1}$ Joining and Welding Research Institute, Osaka University, Ibaraki 567-0047, Japan \\ ${ }^{2}$ Institute of Multidisciplinary Research for Advanced Materials, Tohoku University, Sendai 980-8577, Japan \\ ${ }^{3}$ The Institute of Scientific and Industrial Research, Osaka University, Ibaraki 567-0047, Japan
}

\begin{abstract}
Lotus-type porous metals, whose pores are aligned in one direction by unidirectional solidification, have a unique combination of properties. These are expected as innovative engineering materials with anisotropy of the properties. A reliable joining technology such as welding is required for the industrial application of the lotus-type porous metals as well as processing technology. We have already investigated the melting property of the lotus-type porous copper and magnesium by laser beam irradiation and have pointed out that these materials possessed anisotropy of melting property with the pore direction perpendicular and parallel to the specimen surface, especially remarkable anisotropy was obtained for the lotus-type porous copper owing to the difference of the laser energy absorption to the specimen surface. In this study, three-dimensional finite element calculations of temperature distribution for the lotus-type porous copper as well as the lotus-type porous magnesium under the non-steady-state conditions were performed in order to investigate the effect of the anisotropy of the laser energy absorption comparing with the anisotropy of thermal conductivity inherent to lotus type porous metals. The effect of these factors on the profile of fusion zone by comparing the results of numerical simulation and the experimental observations were discussed. [doi:10.2320/matertrans.47.2248]
\end{abstract}

(Received February 28, 2006; Accepted June 29, 2006; Published September 15, 2006)

Keywords: lotus-type porous copper, lotus-type porous magnesium, laser welding, anisotropy, absorptivity, thermal properties, thermal analysis, finite element method

\section{Introduction}

Porous metals have a unique combination of properties such as light weight, high absorptive capacity of impact energy, permeability to air and to water, sound insulating and vibration damping properties, low thermal conductivity, good electrical insulating property etc. The development of the porous metals and its application for the industrial use are recently extended in the world. Such porous metals can be produced by casting, plating, powder metallurgy and sputter deposition, in which porous metals with higher porosity are called as foamed or cellular structured metals. For example, the foamed aluminum using $\mathrm{TiH}_{2}$ as the foaming agent has a unique combination of properties such as lightweight and high impact energy absorption. ${ }^{1,2)}$ However, the mechanical strength is certainly low mainly due to the stress concentration $^{3)}$ because the pores are spherical and randomly distributed. On the other hand, lotus loot like (lotus-type) porous metals ${ }^{4-19)}$ have higher strength than the conventional porous metals because these pores aligned by the unidirectional solidification. In particular, the lotus-type porous metals are expected as the innovative engineering materials, which yield various anisotropic properties, depending on alignment of the pore growth direction.

For the industrial use of foamed and porous metals as various parts, reliable joining technologies such as welding as well as processing technologies are indispensable. There have been some reports on arc welding of cellular aluminum sandwich plate ${ }^{20)}$ laser welding and arc welding of foamable aluminum, ${ }^{21)}$ arc welding of aluminum-based foam materials, ${ }^{22)}$ laser welding of aluminum foams, ${ }^{23)}$ diffusion bonding of closed-cell aluminum foams, ${ }^{24)}$ and laser based welding of cellular aluminum. ${ }^{25)}$ However, weldability of porous metals with controlled pore direction such as lotus-type porous metals had not been investigated previously. Murakami et al. reported the melting property of the lotus-type porous copper $^{26)}$ and magnesium ${ }^{27)}$ by laser beam irradiation. These results indicated that there was the anisotropy in the penetration depth, especially remarkable anisotropy for the lotus-type porous copper. They suggested that the extraordinary difference in the penetration depth for the lotus-type porous copper may be caused by the notable increase of the effective laser power due to the multiple reflections of the laser beam along the pore wall side in the case of the pore direction parallel to the specimen top surface as well as the high reflectivity of laser beam.

In this study, therefore, three-dimensional finite element calculations of temperature distribution for the lotus-type porous copper with higher laser reflectivity as well as the lotus-type porous magnesium with lower laser reflectivity under the non-steady-state conditions were performed in order to investigate the effect of the anisotropy of the laser energy absorption in comparison with the anisotropy of thermal conductivity inherent to the lotus-type porous metal. The effect of these factors on the profile of fusion zone by comparing the results of numerical simulation and the experimental observations were discussed.

\section{Thermal and Heat Input Modelling of Lotus-Type Porous Metals}

Three-dimensional heat conductive analysis, which takes into account the anisotropy of thermal conductivity, was performed using a commercial FEM code, ABAQUS. The lotus-type porous metal is modeled by an orthotropic material, in which the principal axis for material properties is along the aligned direction of pores. Here, the modeling of thermal properties and heat input are only described briefly. 


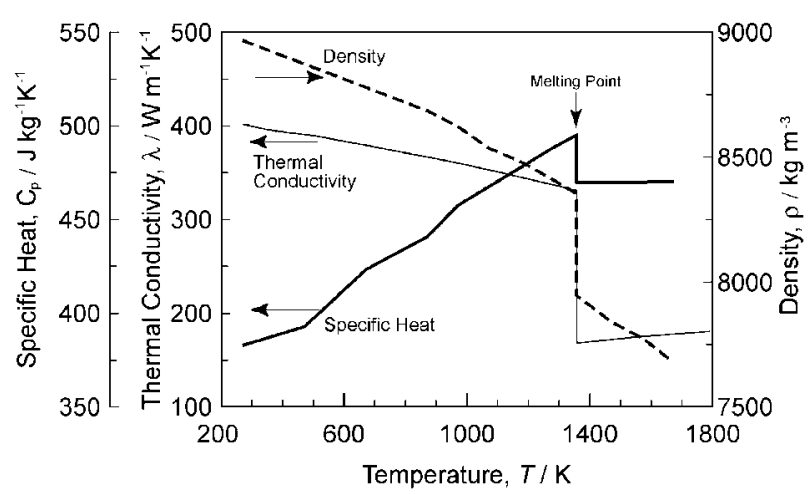

Fig. 1 Temperature dependence of specific heat, thermal conductivity and density of nonporous copper. ${ }^{30,31)}$

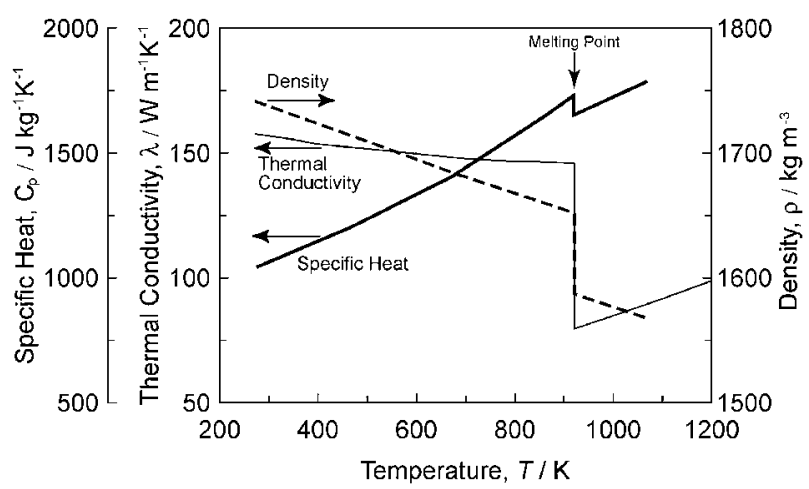

Fig. 2 Temperature dependence of specific heat, thermal conductivity and density of nonporous magnesium. ${ }^{30,31)}$

More detailed description had been introduced in the previous reports. $^{27,28)}$

The thermal analysis was conducted using temperature dependence of material properties. They are the density, specific heat, and thermal conductivity. The equivalent thermal properties of the lotus-type porous metal were calculated from that of nonporous metal by the rule of mixture. ${ }^{29)}$ Assuming the porosity of the lotus-type porous metal is constant with the temperature change, the equivalent density, equivalent specific heat, and equivalent thermal conductivity of the lotus-type porous metal are described by the following equations. ${ }^{27,28)}$

$$
\begin{aligned}
\rho_{e q}(T) & =(1-\varepsilon) \rho_{n}(T), \\
C_{p e q}(T) & =C_{p n}(T), \\
\lambda_{e q}^{\|}(T) & =(1-\varepsilon) \lambda_{n}(T), \\
\lambda_{e q}^{\perp}(T) & =(1-\varepsilon) \cdot(1+\varepsilon)^{-1} \cdot \lambda_{n}(T) .
\end{aligned}
$$

Here, $\rho_{s u b}(T), C_{p \text { sub }}(T)$, and $\lambda_{s u b}^{\operatorname{dir}}(T)$ are the density, specific heat, and thermal conductivity, respectively. The subscript $s u b$ of $e q$ and $n$ implies the equivalent property and the nonporous property, respectively, and the superscript dir of $\|$ and $\perp$ indicates the direction parallel and perpendicular to the unidirectional pores, respectively. $\varepsilon$ is the pore volume content rate. The temperature dependence of the density, specific heat, and thermal conductivity of the nonporous copper and magnesium were shown in Figs. 1 and 2, respectively. ${ }^{30,31)}$

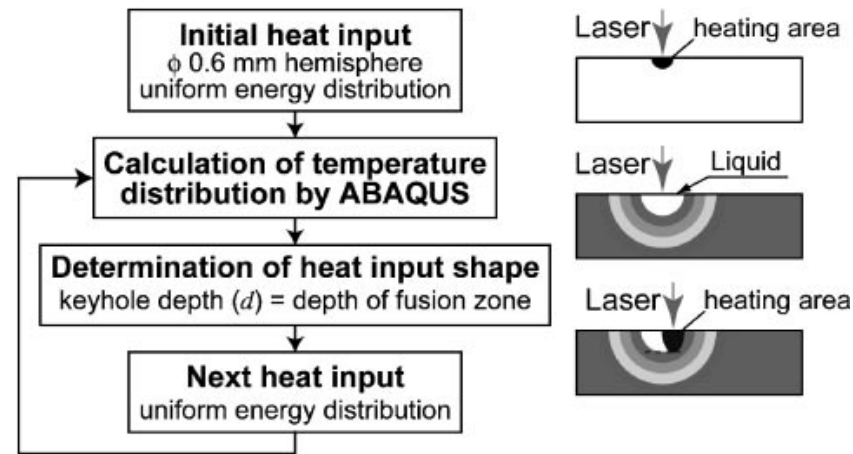

Fig. 3 Flow chart of determination of temperature distribution and shape of heat source.

The heat input to the specimen is generally calculated from the energy supplied at the keyhole. There have been many reports on the distributed heat source models by arc and laser welding, ${ }^{32-39)}$ which was based on Gaussian distribution of heat flux. In this study, however, the growth of the keyhole during the laser welding is only modeled, which is not considered the Gaussian distribution of heat flux. Figure 3 shows the flow chart of the determination of the shape of the heat source and the calculation of the temperature distribution. The model of the heat source assumes a uniform density distribution of energy on the weld pool simulated by a halfspheroid. The shape of the heat source, which changes with time, is defined as the diameter of $0.6 \mathrm{~mm}$ and the depth of $d \mathrm{~mm}$, which is set to the depth of the weld pool at the laser spot. The location and the shape of the heat source are calculated and the depth of weld pool is stored by the ABAQUS user subroutine of DFULX for each increment. Then, the depth of the heat source for the next time increment is updated by the ABAQUS user subroutine of UEXTERNALDB according to the stored depth of weld pool. Here, the volume reduction due to melting the pores, the convection in the weld metal, and the latent heat are neglected for the simplification of the calculation.

The heat conduction on the specimen surface is only considered as the uniform boundary condition. Heat loss due to thermal radiation between the specimen and environment is neglected for the simplification of the calculation. The heat flux on the specimen surface is defined as follows:

$$
q_{s}=\alpha_{s}\left(T_{s}-T_{a}\right),
$$

where $T_{s}$ is the temperature on the specimen surface; $T_{a}$, the ambient temperature; and $\alpha_{s}$, the heat transfer coefficient. In this study, $\alpha_{s}$ is adopted as $1.0 \mathrm{~W} \mathrm{~m}^{-2} \mathrm{~K}^{-1}$, which is the minimum value of air without flow. ${ }^{40)}$

\section{Experimental Procedure}

Lotus-type porous copper was prepared by unidirectional solidification of molten electrolytic copper of $99.99 \%$ purity under a high-pressure mixture of hydrogen $(0.25 \mathrm{MPa})$ and argon $(0.15 \mathrm{MPa})$ gas. ${ }^{9-14)}$ The obtained ingot was $100 \mathrm{~mm}$ in diameter and $100 \mathrm{~mm}$ in height. The average pore diameter and the porosity of specimens were about $0.1 \mathrm{~mm}$ and $30 \%$, respectively. In the same way, the same size ingot of the 

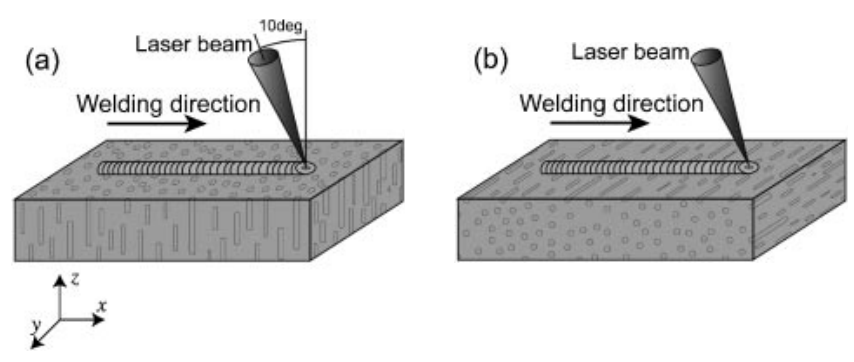

Fig. 4 Schematic views of specimens during laser welding in the porous metal with the pores (a) perpendicular and (b) parallel to the specimen surface.

lotus-type porous magnesium was prepared by unidirectional solidification of molten magnesium of $99.9 \%$ purity under a high-pressure hydrogen gas, which was controlled to be $0.9 \mathrm{MPa} .{ }^{15,16)}$ The average pore diameter and the porosity of specimens were about $0.15 \mathrm{~mm}$ and $35 \%$, respectively. The specimens cut out of the porous ingots with the pore growth directions perpendicular and parallel to the specimen surface using a spark-erosion wire-cutting machine (Brother Industries Ltd., HS-300). The specimen size of porous copper and magnesium were $40 \mathrm{~mm} \times 50 \mathrm{~mm}$ with $4 \mathrm{~mm}$ in thickness and $40 \mathrm{~mm} \times 40 \mathrm{~mm}$ with $1.8 \mathrm{~mm}$ in thickness, respectively.

Figure 4 shows schematic views of the specimens during laser welding. The welding of the specimen was conducted using a Nd:yttrium-aluminum-garnet (Nd:YAG) laser unit with a maximum nominal output power of $3.2 \mathrm{~kW}$ in continuous wave mode. The wavelength of the Nd:YAG laser beam is $1064 \mathrm{~nm}$ and is delivered by using an optical fiber of diameter $0.3 \mathrm{~mm}$. This laser is irradiated the specimen surface by the laser head with the spot diameter of $0.6 \mathrm{~mm}$ and the focal length of $100 \mathrm{~mm}$ at an angle of $80^{\circ}$ to prevent damage to the optics by a reflected laser beam. Argon was used as a shielding gas with a flow rate of 30 $l / \mathrm{min}$. The welding speed was controlled in $0.2 \mathrm{~m} / \mathrm{min}$ with the laser power of $3.2 \mathrm{~kW}$ for the copper specimen and 5 $\mathrm{m} / \mathrm{min}$ with the laser power of $1.0 \mathrm{~kW}$ for the magnesium specimen, respectively. The cross sections of the welded specimen were observed with an optical microscopy in order to determine the profile of the weld fusion zone.

\section{Comparison between Experimental Results and Cal- culation of Weld Fusion Profile}

Thermal analyses under the non-steady-state conditions for the lotus-type porous copper and magnesium were carried out and the temperature distribution was evaluated by the three-dimensional finite element analysis. It was supposed that the size and the welding speed for the copper specimen are $40 \mathrm{~mm}(x) \times 20 \mathrm{~mm}(y) \times 4 \mathrm{~mm}(z)$ and $0.2 \mathrm{~m} / \mathrm{min}$, respectively, and $20 \mathrm{~mm}(x) \times 10 \mathrm{~mm}(y) \times 1.8 \mathrm{~mm}(z)$ and $5 \mathrm{~m} / \mathrm{min}$, respectively for the magnesium specimen. The initial specimen temperature is $298 \mathrm{~K}$ and the initial depth of heat source $d$ is $0.3 \mathrm{~mm}$. The ambient temperature $T_{a}$ is assumed to the constant value of $298 \mathrm{~K}$. The analysis region is $y>0$ due to space symmetry because the welding direction is along the $x$-axis. The unit element size for the copper specimen is $1.0 \mathrm{~mm}(x) \times 1.0 \mathrm{~mm}(y) \times 0.5 \mathrm{~mm}(z)$ and the element size for the magnesium specimen is 0.25 $\mathrm{mm}(x) \times 0.25 \mathrm{~mm}(y) \times 0.3 \mathrm{~mm}(z)$. The porosity of the copper specimen and the magnesium specimen are $35 \%$ and $30 \%$, respectively. In the case for the direction of the pore growth is perpendicular to the specimen top surface, the thermal conductivities are $\lambda_{z}(T)=\lambda_{e q}^{\|}(T)$ and $\lambda_{x}(T)=$ $\lambda_{y}(T)=\lambda_{e q}^{\perp}(T)$, which is calculated from eq. (2). On the other hand, the thermal conductivity are $\lambda_{y}(T)=\lambda_{e q}^{\|}(T)$ and $\lambda_{x}(T)=\lambda_{z}(T)=\lambda_{e q}^{\perp}(T)$ for the pore parallel to the specimen top surface. Here the subscript $x, y, z$ means the thermal conductivities along with the $x$-, $y$-, and $z$-axis, respectively.

In order to compare the experimental fusion profile, the calculated profile of the weld metal is estimated by the cross section of the maximum temperature distribution at half of the $x$ direction. In other words, the region exceed the melting temperature on this distribution implies the fusion zone.

Figure 5 shows the maximum temperature distributions in the copper specimen with the pores perpendicular and parallel to the specimen top surface together with the bead appearance and the cross section of experimental results. ${ }^{26)}$ Moreover, the maximum temperature distributions in the magnesium specimen also shown in Fig. 6 together with the bead appearance and the cross section of experimental results. ${ }^{27)}$ Here, the absorbable laser power at the specimen top surface is assumed that the most similar results between the experimental fusion depth and the calculated ones for the parallel direction in Fig. 5 and in Fig. 6, respectively. That is to say, the difference of the laser energy absorption did not take into account for these calculated results. Compared between calculated results with the absorbable laser power of $0.18 \mathrm{~kW}$ or $0.54 \mathrm{~kW}$ and experimental results, the depth of the fusion zone was comparatively similar in the parallel direction for the both materials. However, in the perpendicular direction, the profile of the fusion zone is different between the calculated result and the experimental ones, especially, for the copper specimen. Therefore, the absorbable laser power at the specimen top surface for the perpendicular direction is re-estimated so that the most similar results between the experimental fusion depth and the calculated ones can be obtained by taking into account the difference of the laser energy absorption between the perpendicular case and the parallel one. The maximum temperature distributions for the perpendicular direction in the both materials are also shown in Figs. 5 and 6. The depth of calculated profiles and the experimental ones were similar in the both materials.

The effective absorptivity of laser power for these four cases can be estimated from the ratio of nominal laser power for experiments and the absorbable laser power for calculations. It is $10.3 \%$ and $5.6 \%$ for the perpendicular and the parallel directions of the copper specimen, respectively, and $72 \%$ and $54 \%$ for the perpendicular and the parallel directions of the magnesium specimen, respectively. Therefore, the effective absorptivity of the lotus-type porous copper for the perpendicular direction is twice larger than that for the parallel one, but there is not so much difference between two directions for the lotus-type porous magnesium. The reason is considered as follows; the laser beam mostly reflects on the copper surface due to the high reflectivity of copper, about $95 \%$ at room temperature. ${ }^{41,42)}$ In the perpen- 


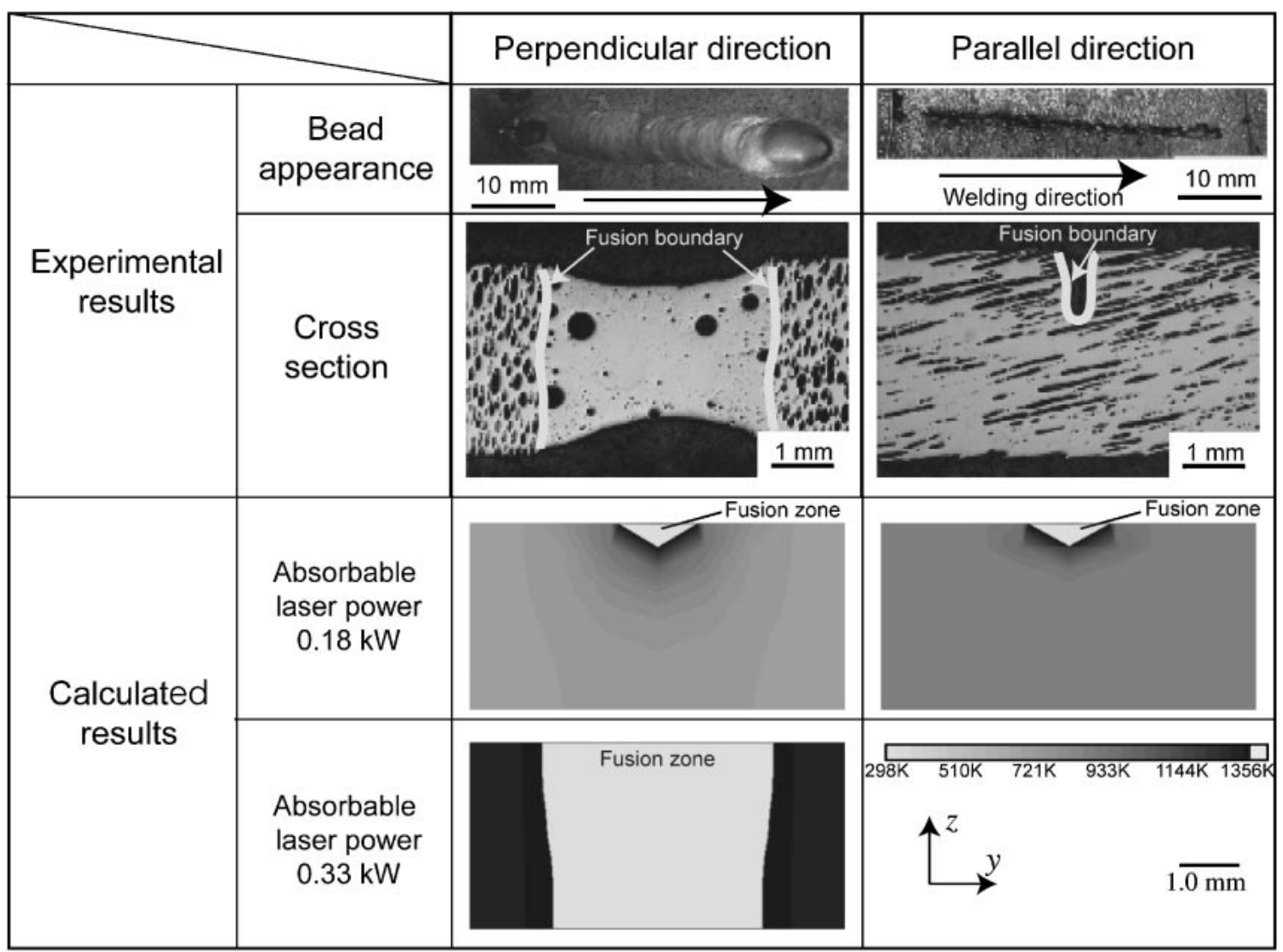

Fig. 5 Comparison between calculated maximum temperature distributions in the cross-section at half of the $x$ direction and macrostructure of the corresponding laser welded specimen with the nominal laser power of $3.2 \mathrm{~kW}$ for the lotus-type porous copper with the welding speed of $0.2 \mathrm{~m} / \mathrm{min}^{26}$ )

dicular direction, a part of laser beam deeply penetrates into open pores because the growth direction of original pore nearly equals to the incident direction of the laser. In this case, the multiple reflections of the laser beam along the pore wall side extraordinarily increase the absorbable laser power. The similar phenomenon had found in the welding for the high-density energy heat source and this is called "Wallfocusing Effect" ${ }^{43)}$ On the other hand, as the reflectivity of magnesium is $74 \%$ at room temperature, ${ }^{41)}$ a part of laser beam penetrates into open pores but almost laser power is absorbed within a few reflections in the perpendicular direction. In this case, therefore, this phenomenon slightly increases the absorbable laser power.

Compared between calculated and experimental profiles of weld fusion zone, however, the calculated width of the weld metal is slightly larger than the experimental result. The reason is the simplification of analysis, which is not considered the distributed energy density for the heat source, the convection in the weld metal, and the heat transfer by the latent heat due to the melting and vaporization of the specimen. These factors, however, not so influence significantly on the anisotropic profile of weld fusion zone than the difference of the laser energy absorption because these factors are the isotropic phenomena. Accordingly, the extraordinary difference between the weld fusion zone of the lotus-type porous copper with the pores parallel to the specimen top surface and perpendicular one is mainly controlled by the difference of the laser energy absorption on the specimen top surface, and the relatively slight difference of profile for the lotus-type porous magnesium is mainly controlled by anisotropy of thermal conductivity.

\section{Conclusions}

Three-dimensional finite element calculations of temperature distribution for the lotus-type porous copper with higher laser reflectivity as well as the lotus-type porous magnesium with lower laser reflectivity under the nonsteady-state conditions were performed in order to investigate the effect of the anisotropy of the laser energy absorption. The effect of this factor on the profile of fusion zone was discussed by comparing the results of numerical simulation and the experimental observations.

The effective absorptivity of laser power has been estimated from the ratio of nominal laser power for experiments and the absorbable laser power for calculations. The effective absorptivity of the lotus-type porous copper for the perpendicular direction is 2 times larger than that for the parallel one, but there is not so much difference between two directions for the lotus-type porous magnesium. These results are due to the difference of the reflectivity of laser beam between copper and magnesium.

It has been concluded that the profile difference between the weld fusion zone of the lotus-type porous copper with the pores parallel to the specimen top surface and perpendicular one is mainly controlled by the difference of the laser energy 


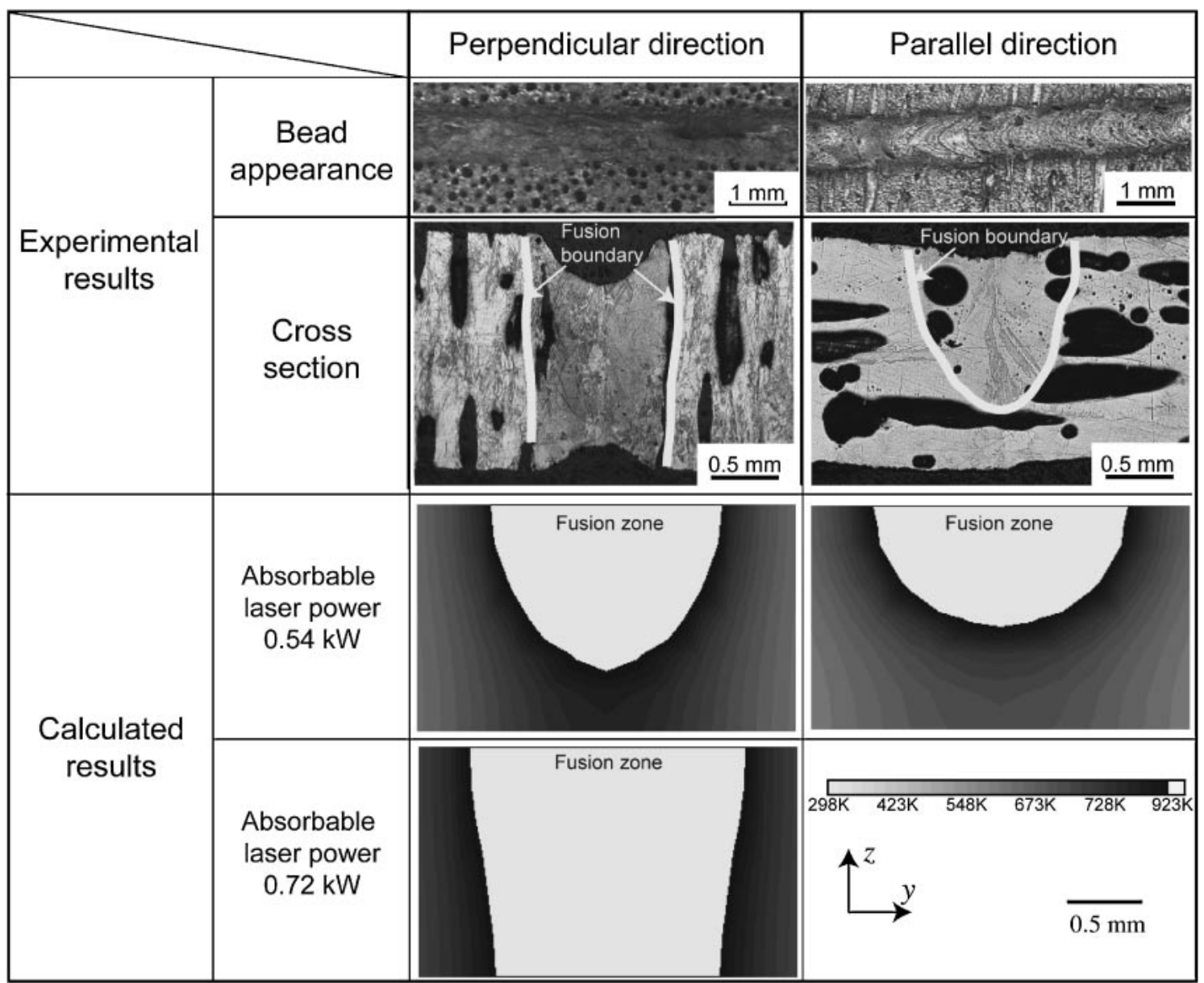

Fig. 6 Comparison between calculated maximum temperature distributions in the cross-section at half of the $x$ direction and macrostructure of the corresponding laser welded specimen with the nominal laser power of $1.0 \mathrm{~kW}$ for the lotus-type porous magnesium with the welding speed of $5 \mathrm{~m} / \mathrm{min}^{27}$ )

absorption on the specimen top surface and the profile difference for the lotus-type porous magnesium is mainly controlled by the anisotropy of thermal conductivity.

\section{REFERENCES}

1) B. Zettl, H. Mayer and S. E. Stanzl-Tschegg: Int. J. Fatigue 23 (2001) 565-573.

2) S. Ramachandra, P. Sudheer Kumar and U. Ramamurty: Scr. Mater. 49 (2003) 741-745.

3) H. P. Degischer and B. Kriszt: Handbook of Cellular Metals: Production, Processing, Applications, (Wiley-VCH Verlag $\mathrm{GmbH}$, Weinheim, 2002).

4) V. I. Shapovalov: Porous and Cellular Materials for Structural Applications, ed. by D. S. Schwartz, D. S. Shih, A. G. Evans and H. N. G. Wadley, (MRS, Warrendale, USA, 1998) 281-290.

5) H. Nakajima: Mater. Integration 12 (1999) 37-44.

6) H. Nakajima: The Production \& Tech. 51 (1999) 60-62.

7) H. Nakajima: Boundary 15 (1999) 9-11.

8) H. Nakajima: J. High Temp. Soc. 26 (2000) 95-100.

9) H. Nakajima: Bull. The Iron and Steel Institute of Japan 6 (2001) 701707.

10) H. Nakajima, S. K. Hyun, K. Ohashi, K. Ota and K. Murakami: Colloids Surf. A 179 (2001) 209-214.

11) S. K. Hyun, K. Murakami and H. Nakajima: Mater. Sci. Eng. A 299 (2001) 241-248.

12) S. K. Hyun and H. Nakajima: Mater. Sci. Eng. A 340 (2003) 258-264
13) K. Ota, K. Ohashi and H. Nakajima: Mater. Sci. Eng. A 341 (2003) 139-143.

14) T. Ogushi, H. Chiba, H. Nakajima and T. Ikeda: J. Appl. Phys. 95 (2004) 5843-5847.

15) T. Ikeda and H. Nakajima: J. Jpn. Foundry Eng. Soc. 74 (2002) 812816.

16) T. Ikeda, H. Hoshiyama and H. Nakajima: J. Jpn. Inst. Light Met. 54 (2004) 388-393.

17) S. K. Hyun and H. Nakajima: Mater. Trans. 43 (2002) 526-531.

18) S. K. Hyun, T. Ikeda and H. Nakajima: Sci. Technol. Adv. Mater. 5 (2004) 201-205.

19) M. Tane, T. Ichitsubo, H. Nakajima, S. K. Hyun and M. Hirano: Acta Mater. 52 (2004) 5195-5201.

20) U. Dilthey and M. Kessel: Schwei en und Schneiden, DVS-Berichte 220 (2002) 216-218.

21) Th. Bölinghaus and W. Bleck: Cellular Metals and Metal Foaming Technology, (Verlag MIT Publishers, Bremen, 2001) 495-500.

22) A. G. Pogibenko, V. Y. Konkevich, L. A. Arbuzova and V. I. Ryazantsev: Weld. Int. 15 (2001) 312-316.

23) T. Bernard, J. Burzer and H. W. Bergmann: J. Mater. Process. Tech. 115 (2001) 20-24.

24) K. Kitazono, A. Kitajima, E. Sato, J. Matsushita and K. Kuribayashi: Mater. Sci. Eng. A 327 (2002) 128-132.

25) H. Haferkamp, J. Bunte, D. Herzog and A. Ostendorf: Sci. Technol. Weld. Joining 9 (2004) 65-71.

26) T. Murakami, K. Nakata, T. Ikeda, H. Nakajima and M. Ushio: Mater. Sci. Eng. A 357 (2003) 134-140.

27) T. Murakami, T. Tsumura, T. Ikeda, H. Nakajima and K. Nakata: Submitted to Mater. Sci. and Eng. A. 
28) T. Tsumura, T. Murakami, S. K. Hyun, H. Nakajima and K. Nakata: Mater. Sci. Forum 502 (2005) 499-504.

29) F. L. Matthews, G. A. O. Davies, D. Hitchings and C. Soutis: Finite Element Modelling of Composite Materials and Structures, (Woodhead Publishing Ltd., Cambridge, 2000) p. 9.

30) Y. S. Touloukian, R. W. Powell, C. Y. Ho and P. G. Klemens: Thermophysical Properties of Matter: Vol. 1; Thermal Conductivity: Metallic Elements and Alloys, (IFI/Plenum Data Corp., New York, 1970) p. 207.

31) T. Lyman, H. E. Boyer, P. M. Unterweiser, J. E. Foster, J. P. Hontas and H. Lawton: Metals Handbook, 8th ed.; Vol. 1: Properties and Selection of Metals, (American Society for Metals, Ohio, 1967) p. 1214.

32) J. Mazumder and W. M. Steen: J. Appl. Phys. 51 (1980) 941-947.

33) J. Goldak, A. Chakravarti and M. Bibby: Metall. Trans. B 15 (1984) 299-305.

34) I. Miyamoto, H. Maruo and Y. Arata: Laser Processing: Fundamentals, Applications, and Systems Engineering, (SPIE Vol. 668, Washington,
USA, 1986) 11-18.

35) T. Zacharia, S. A. David, J. M. Vitek and T. Devroy: Metall. Trans. A 20 (1989) 957-967.

36) P. Michaleris and A. DeBiccari: Weld. J. 76 (1997) 172s-181s.

37) G. Yu, K. Masubuchi, T. Maekawa and N. M. Patrikalakis: Transactions of the ASME, J. Manuf. Sci. Eng. 123 (2001) 405-410.

38) W. S. Chang and S. J. Na: J. Mater. Process. Tech. 120 (2002) 208-214.

39) S. A. Tsirkas, P. Papanikos and Th. Kermanidis: J. Mater. Process. Tech. 134 (2003) 59-69.

40) Dictionary of Physics Editors Committee, Dictionary of Physics, reduced-size ed., (Baifukan Co. Ltd., Tokyo, 1984) p. 1537.

41) E. A. Brandes: Smithells Metal Reference Book, 6th ed., (Butterworths, London, 1983) p. 17-7.

42) H. Watanabe, M. Susa and K. Nagata: Metall. Matel. Trans. A 28 (1997) 2507-2513.

43) Y. Arata: Transactions of JWRI 2 (1973) 119-120 